\title{
Kernos
}

Revue internationale et pluridisciplinaire de religion grecque antique

$28 \mid 2015$

Varia

\section{Topographie sacrée et structure narrative chez}

Pausanias

Du Dipylon à l'Académie

Jean Vanden Broeck-Parant

\section{(2) OpenEdition \\ Journals}

Édition électronique

URL : http://journals.openedition.org/kernos/2335

DOI : 10.4000/kernos.2335

ISSN : 2034-7871

Éditeur

Centre international d'étude de la religion grecque antique

\section{Édition imprimée}

Date de publication : 1 octobre 2015

Pagination : 155-173

ISBN : 978-2-87562-055-2

ISSN : 0776-3824

Référence électronique

Jean Vanden Broeck-Parant, «Topographie sacrée et structure narrative chez Pausanias », Kernos [En ligne], 28 | 2015, mis en ligne le 01 octobre 2017, consulté le 24 février 2021. URL : http://

journals.openedition.org/kernos/2335; DOI : https://doi.org/10.4000/kernos.2335

Ce document a été généré automatiquement le 24 février 2021.

Kernos 


\title{
Topographie sacrée et structure narrative chez Pausanias
}

\author{
Du Dipylon à l'Académie
}

Jean Vanden Broeck-Parant

Je remercie les Prof. Didier Viviers et Alain Martin pour leurs corrections et les discussions que nous avons eues, Constanze Graml, pour ses précieux commentaires et Dorian Vanhulle, pour sa relecture attentive.

1 Dans le premier livre de la Périégèse, Pausanias décrit l'Attique. Il commence par les ports d'Athènes, le Pirée et le Phalère, puis emprunte les routes qui menaient de ces ports à la ville même, avant de décrire la cité intra muros. Il entreprend ensuite la description des régions se trouvant à l'extérieur des murs d'Athènes ${ }^{1}$ :

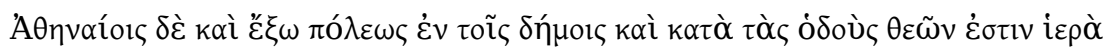

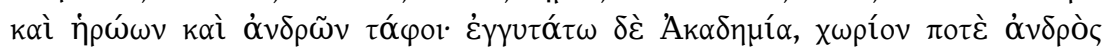

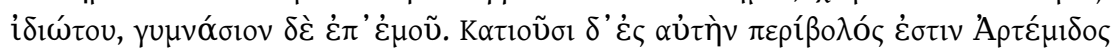

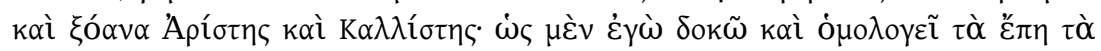

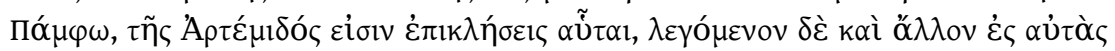

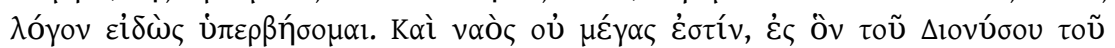

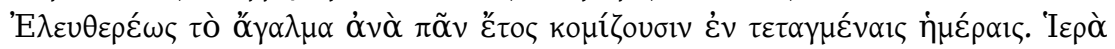

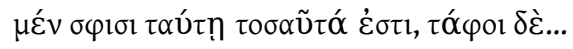

Les Athéniens ont aussi, à l'extérieur de la cité, dans les dèmes et le long des routes, des sanctuaires de dieux et des tombes de héros et d'hommes. Tout près se trouve l'Académie qui était autrefois le domaine d'un particulier et est aujourd'hui un gymnase. En y descendant, on rencontre le peribolos d'Artémis et les xoana d'Aristè et de Kallistè ; comme je le crois - et les vers de Pamphos confirment mon opinion, ce sont des surnoms d'Artémis. Je connais une autre histoire les concernant, que je laisserai ici de côté. Il y aussi un petit naos, où l'on amène chaque année à certains jours la statue de Dionysos Eleuthereus. Tels sont les sanctuaires qui se trouvent là ; quant aux tombeaux, ...

2 Ainsi Pausanias commence-t-il sa description de l'Athènes extra muros, dans la région $\mathrm{du}$ Kerameikos $^{2}$ et de l'Académie. Après avoir évoqué les sanctuaires, le Périégète poursuit en énumérant les différents tombeaux que l'on pouvait rencontrer en parcourant la prestigieuse voie qui menait à l'Académie. 
3 L'objectif de cet article est de proposer une localisation plus précise du naos de Dionysos Eleuthereus mentionné par Pausanias. À cette fin, on fera d'abord le point sur ce que l'on sait de la situation du sanctuaire d'Artémis. Cette localisation est en effet nécessaire pour aborder une lecture approfondie du passage du Périégète, laquelle permettra de resserrer le champ d'investigation pour situer le naos de Dionysos ${ }^{3}$.

D'un point de vue strictement archéologique, les recherches sont pratiquement au point mort depuis l'entre-deux-guerres, pour la raison que l'on connait : l'urbanisation galopante d'Athènes, en particulier à partir des années $1950^{4}$, a mis fin à tout espoir d'un jour procéder à une fouille étendue dans la région ${ }^{5}$. Pour cette raison, le sanctuaire d'Artémis n'a pratiquement plus fait l'objet de discussions depuis l'article d'A. Philadelpheus publié en 1927, dans lequel il disait l'avoir probablement localisé. Nous verrons quel crédit peut être accordé à cette localisation. Le cas du temple de Dionysos est autrement plus compliqué, puisqu'aucun vestige n'a pu encore lui être attribué à ce jour.

\section{Le sanctuaire d'Aristè et de Kallistè au Kerameikos}

5 Le Périégète ne parle pas d'un "sanctuaire d'Artémis Aristè et Kallistè " ${ }^{6}$, mais du peribolos (l'enceinte) d'Artémis et des xoana, c'est-à-dire des statues, d'Aristè et de Kallistè. Néanmoins, puisqu'il explique aussitôt qu'Aristè et Kallistè sont deux épithètes d'Artémis, on en a logiquement déduit que les statues se trouvaient dans ou à proximité de l'enceinte et que celle-ci devait être consacrée aux deux divinités. Cette hypothèse peut être appuyée par l'analyse que fait V. Pirenne-Delforge du terme peribolos chez Pausanias: "Le choix du terme relève d'un souci d'exactitude dans la description puisqu'il fait référence à une réalité topographique tangible; il articule également la présentation des diverses composantes d'un sanctuaire. (...) Le propos passe donc graduellement du générique d'un lieu sacré au particulier des statues de culte. $\gg^{7}$ Est-ce à dire que les xoana d'Aristè et Kallistè étaient compris dans une enceinte consacrée à ces deux divinités et à elles seules ? Les différentes découvertes faites dans les environs permettent d'aller dans ce sens.

6 En 1896, au cours de fouilles à l'angle des rues Salaminos et Piréos ${ }^{8}$, A. Oikonomou rencontrait probablement le péribole lorsqu'il mit au jour une surface pavée de 11 mètres de large, des restes de fondations en blocs quadrangulaires de pôros ainsi qu'une inscription ${ }^{9}$ datée de $235 / 4$ av. J.-C. et mentionnant un "prêtre de Kallistè »

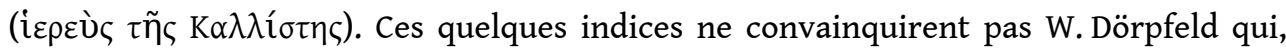
l'année même des fouilles, exprimait déjà des réserves quant à l'identification des vestiges, estimant nécessaire d'établir au préalable le plan au sol de la construction ${ }^{10}$.

7 En 1922, A. Philadelpheus dissipa ces doutes en mettant au jour, au numéro 11 de la rue Plataiôn (soit tout près des fouilles d'A. Oikonomou), des restes de construction formant un angle et présentant une technique et des matériaux similaires à ceux qui avaient été découverts vingt-six ans auparavant ${ }^{11}$. Surtout, de nouvelles inscriptions et des reliefs votifs (deux bases dédiées à Kallistè, datées du IV ou du III ${ }^{\mathrm{e}}$ siècle avant J.-C. ${ }^{12}$, un relief en marbre du III $^{\mathrm{e}}$ siècle avant J.-C. figurant deux seins et dédié à Kallistè̀ ${ }^{13}$, trois reliefs votifs avec des représentations d'anatomie féminine et un relief votif avec une déesse tenant une torche, de date similaire ${ }^{14}$ ) venaient renforcer l'hypothèse selon 
laquelle le péribole des Artémis Aristè et Kallistè se trouvait dans le voisinage ; à tout le moins, celui de Kallistè.

8 Aristè était en effet encore absente des inscriptions. Il fallut attendre 1938 pour qu'apparaisse, à l'Agora, une dédicace à Aristè et Kallistè ${ }^{15}$. Pour la première fois, les deux épithètes étaient réunies indépendamment de Pausanias, attribuant un crédit renouvelé aux informations données par le Périégète. J.H. Oliver ne devait pas avoir connaissance de cette dédicace qu'il ne mentionna pas lorsqu'il restitua en 1941

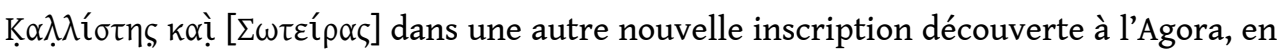
se fondant sur une hypothèse de W.S. Ferguson ${ }^{16}$. Il prit cependant soin de préciser qu'il

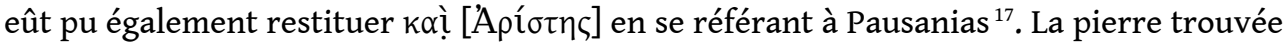
en 1938 dut attendre 1959 pour être publiée par A.G. Woodhead, lequel revenait alors sur l'interprétation de J.H. Oliver pour insister sur la probabilité, désormais renforcée, de la restitution [Apíornc] ${ }^{18}$.

9 Ces deux découvertes - les fouilles d'A. Philadelpheus et la dédicace à Aristè et Kallistè - laissent peu de place au doute : il y avait bien un lieu de culte dédié à la fois à Aristè et à Kallistè ${ }^{19}$ au Kerameikos et on peut le localiser selon toute vraisemblance à l'endroit des fouilles d'A. Philadelpheus et A. Oikonomou ou, tout au moins, dans les environs immédiats ${ }^{20}$. Cette première conclusion nous donne un point d'ancrage important par rapport auquel il est dès lors possible de mieux situer le naos de Dionysos Eleuthereus.

\section{Localiser le temple de Dionysos Eleuthereus à l'aide de la structure narrative?}

10 Si les sources sont relativement abondantes en ce qui concerne les Artémis du Kerameikos, le Dionysos Eleuthereus de Pausanias reste évanescent. Le Périégète évoque très brièvement son naos qui, dit-il, «n'est pas grand» et dont la proximité dans le texte avec le peribolos des Artémis Aristè et Kallistè a conduit certains chercheurs à penser que les deux sanctuaires devaient être également proches d'un point de vue topographique, sinon pratiquement contigus. À l'inverse, on a parfois situé le naos de Dionysos Eleuthereus "à l'Académie »" suivant, sans doute, ce passage de Philostrate ${ }^{22}$ :

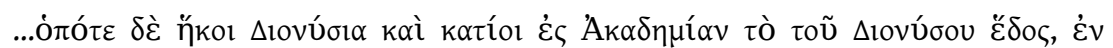

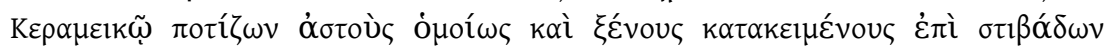

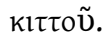

Et lorsque les Dionysies revenaient et que l'image de Dionysos descendait à l'Académie, il (Hérode Atticus) offrait à boire aux Athéniens et aux étrangers qui, au Kerameikos, étaient couchés sur des feuilles de lierre.

Philostrate et Pausanias sont les seuls auteurs qui nous donnent des informations de nature topographique sur le naos de Dionysos Eleuthereus, encore que chez Philostrate le lieu de culte ne soit pas mentionnée ${ }^{23}$. Les deux auteurs utilisent rigoureusement les

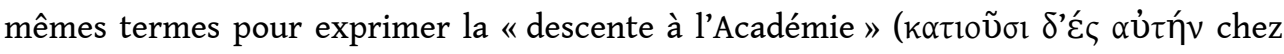

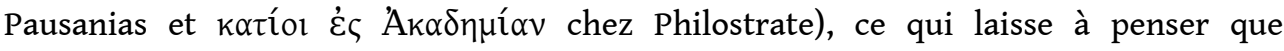
Philostrate s'est peut-être inspiré directement de Pausanias ou, plus probablement, qu'il s'agissait d'une expression consacrée. Mais surtout, la similitude des formules utilisées laisse croire que les parcours décrits par Pausanias et Philostrate étaient, sinon identiques, du moins très comparables et, a fortiori, que l'un et l'autre évoquent le 
même rituel. La formule n'implique absolument pas, en tout cas, que le petit temple de Dionysos Eleuthereus se soit trouvé à l'Académie même.

Nous voudrions montrer, dans les lignes qui suivent, qu'il est possible d'être plus précis en se fondant sur deux éléments en particulier: la localisation du sanctuaire des Artémis Aristè et Kallistè, établie plus haut, et la structure narrative adoptée par Pausanias dans le cadre du passage qui nous intéresse.

Avant de poursuivre notre développement, il paraît ici opportun de situer le naos dont parle Pausanias dans les contextes historique et rituel du culte dans lequel il s'insérait et que l'on connaît relativement bien. Le culte de Dionysos Eleuthereus aurait été introduit à Athènes par un certain Pegasos ${ }^{24}$, sorte d'émissaire du culte originaire d'Éleuthères à l'époque du roi légendaire d'Athènes Amphiktyon, d'après Pausanias ${ }^{25}$. On considère généralement que c'est sous Pisistrate que la fête acquit une réelle importance, une idée récemment battue en brèche par D. Paleothodoros, qui suggère que l'invention des Dionysies urbaines (fêtes en l'honneur de Dionysos Eleuthereus) pourrait plutôt dater de la fin $\mathrm{du} \mathrm{VI}^{\mathrm{e}}$ siècle, dans le contexte de la démocratie naissante ${ }^{26}$. Les festivités ont dû évoluer au cours des siècles ${ }^{27}$, mais on possède des inscriptions qui y font référence jusqu'au début du $\mathrm{I}^{\mathrm{er}}$ siècle avant $\mathrm{J}$.-C. Au $\mathrm{II}^{\mathrm{e}}$ siècle après J.-C., Pausanias évoque encore le transport de la statue du dieu jusqu'à son petit temple, qui a lieu chaque année ${ }^{28}$. Ces indices attestent donc au moins six siècles d'existence.

Avant le début des festivités proprement dites, la statue en bois du dieu, le xoanon, était transportée depuis le temple à proximité du théâtre ${ }^{29}$ jusqu'à son eschara ${ }^{30}$. Il a été généralement considéré que celui-ci se trouvait au même endroit que le petit naos évoqué par Pausanias ${ }^{31}$, mais rien ne permet de le prouver. Il est d'ailleurs envisageable que l'eschara mentionnée par les inscriptions soit celle qui se trouvait à l'Agora, à proximité de l'autel des Douze Dieux ${ }^{32}$. Si tel est bien le cas, il faut alors ajouter un arrêt supplémentaire, au naos, entre le départ du théâtre et l'arrivée à l'eschara. Ensuite avait lieu (probablement après un sacrifice et des chants) l'eisagôgê, l'introduction du dieu à Athènes. Ce trajet se faisait vraisemblablement de nuit puisqu'il était éclairé de torches et aboutissait au théâtre ou au temple voisin. Cette première étape est à distinguer de la pompê, la procession proprement dite, qui avait lieu, semble-t-il, le lendemain matin. Après un arrêt à l'Agora, celle-ci se terminait au temple intra muros, où le sacrifice d'un taureau constituait un climax. Les concours dramatiques pouvaient alors commencer, sans doute dès le lendemain, pour une durée de trois jours ${ }^{33}$.

N.T. Arrington a relevé une série d'éléments militaires qui ponctuent les célébrations : transport de la statue de culte par des éphèbes (jeunes hommes en service militaire), libations préalables aux représentations effectuées par les stratèges, tributs des alliés exposés sur la scène, présentation des orphelins de guerre passés à l'âge adulte entre autres. Il en déduit que: "some of these additions (...) only make sense if the extramural temple [celui de Dionysos Eleuthereus] was situated in the demosion sema $»^{34}$. La remarque suffirait à elle seule à expliquer pourquoi le temple de Dionysos Eleuthereus ne pouvait pas se trouver à l'Académie même, mais c'est dans le récit de Pausanias que l'on en trouve la meilleure preuve.

Si l'on considère que ce dernier rapporte tous les monuments dans l'ordre sans distinction d'aucune sorte, alors il faut situer le temple de Dionysos entre le sanctuaire d'Artémis et le tombeau de Thrasybulle. Cette lecture pose cependant un problème majeur, à savoir que le Périégète ne mentionnerait pas la moindre tombe, pas le 
moindre monument, quel qu'il soit, avant d'évoquer le péribole d'Artémis. Or de nombreuses tombes ont été mises au jour dans le parc du Céramique entre les murs de la ville et l'emplacement probable du sanctuaire d'Artémis. Deux explications ont été proposées pour rendre compte de cette absence chez Pausanias.

La première option est qu'une grande partie de ce qui se trouvait au Kerameikos aurait été recouverte par une épaisse couche de sable à l'époque de Pausanias. Le phénomène, qui est attesté par les fouilles archéologiques, se conjugue avec un événement de la fin du Iv siècle avant J.-C. : suite à la bataille de Chéronée, on se serait hâté de déplacer les monuments funéraires pour renforcer les murs de la ville ${ }^{35}$. On ajoutera que la région avait subi deux destructions majeures avant l'époque du Périégète avec Philippe V de Macédoine ${ }^{36}$ puis Sylla ${ }^{37}$. Cependant, il est difficile de croire que la région du Kerameikos soit restée totalement déserte après ces destructions. Il est même envisageable que certaines restaurations aient été opérées, comme cela a été suggéré pour une stèle mentionnée par Pausanias (I, 29, 11) ${ }^{38}$.

Une deuxième option est que Pausanias aurait emprunté une route parallèle à la voie principale avant de rejoindre celle-ci à hauteur du temple d'Artémis ${ }^{39}$. Un argument en faveur de cette hypothèse est que Pausanias ne dit pas explicitement qu'il part du Dipylon; il a donc pu quitter les murs de la ville à partir d'une porte proche. On peut toutefois opposer trois arguments. D'abord, Pausanias, s'il effectue un tel mouvement, ne l'exprime pas; au contraire, il dit à deux reprises «le long de cette route » (sans aucun doute possible, la route principale vers l'Académie), la première fois après avoir déjà cité quelques tombeaux. Ensuite, cette solution n'explique pas pourquoi Pausanias reste silencieux car, s'il a pris une autre route, il y aura certainement vu d'autres choses dignes d'être mentionnées ${ }^{40}$. Dans le cas contraire enfin, il paraît étrange de vouloir prêter à Pausanias l'intention de prendre une route sur laquelle il n'y aurait rien à voir et dont il n'y aurait rien à dire.

Ces hypothèses partagent le postulat que les tombes mentionnées par Pausanias se trouvaient après les sanctuaires d'Artémis et de Dionysos sur la route qui menait à l'Académie. Cependant, un tel postulat repose uniquement sur l'ordre dans lequel sont mentionnés les monuments ${ }^{41}$ et fait abstraction du texte lui-même. Il néglige en particulier une structuration ostensible du discours, et donc de l'espace, annoncée de manière très claire par le Périégète avant même d'entamer sa description des

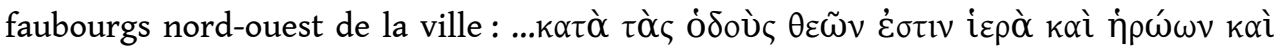
$\dot{\alpha} v \delta \rho \tilde{\omega} v \tau$ นó $\varphi \circ \mathrm{l}(\mathrm{I}, 29,2)$

Ainsi il y a les dieux, les héros et les hommes, selon une hiérarchie qui sera récurrente dans la Périégèse. Le caractère programmatique de cette phrase a déjà été noté par V. Pirenne-Delforge ${ }^{42}$, qui en étend l'exploitation à l'ensemble de l'œuvre de Pausanias, ainsi que par L. Beschi et D. Musti ${ }^{43}$, sans que ces derniers en tirent d'éventuelles conséquences d'un point de vue topographique. Il est cependant possible d'aller plus loin. Cette indication de la manière dont le Périégète organise sa sélection et ses descriptions trouve en effet des applications d'ordre narratif à différents endroits de son œuvre, et en particulier, de manière très claire, aussitôt après cette introduction programmatique. Ainsi, dans le passage qui concerne le faubourg nord-ouest d'Athènes, quelques formules utilisées par le Périégète fixent le cadre topographique et narratif de la séquence.

21 Pausanias commence par donner l'indication géographique $\tilde{\varepsilon} \xi \omega \pi$ ró $\lambda \varepsilon \omega \varsigma$ (I, 29, 2), « en dehors de la cité », que l'on peut comprendre sans peine comme "à l'extérieur de 
l'enceinte de la cité ». Ensuite, et avant d'entrer dans les détails, Pausanias donne une

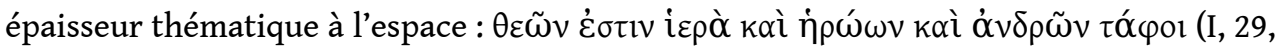
2). Il y a, en dehors de la ville, d'une part des sanctuaires pour les dieux et, d'autre part, des tombes de héros et d'hommes. Cette assertion n'a, jusqu'ici en tout cas, aucune valeur topographique; si temples et tombes sont bien séparés dans l'esprit de Pausanias, ils ne le sont pas nécessairement dans la réalité.

Pausanias annonce ensuite quel sera le point de focalisation de sa description:

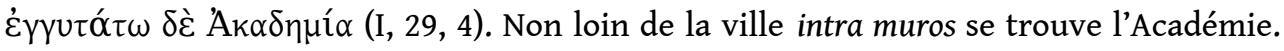
S'il ne le dit pas explicitement à ce moment, par la suite il compartimentera toujours

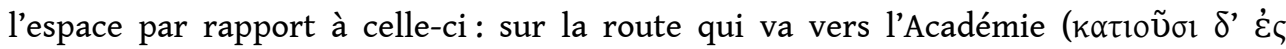

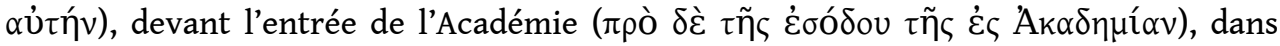

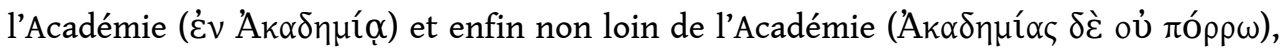
avant de passer à la description du reste de l'Attique. C'est la première partie de cette description, « sur la route qui va vers l'Académie ", qui nous intéressera ici.

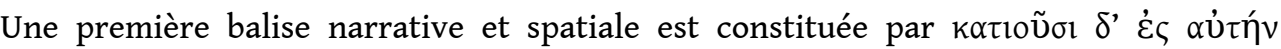
(1.29.4). Pausanias ne dit pas, en fait, «sur la route vers l'Académie» mais «en y descendant ", ce qui ne revient pas exactement au même : il a pu emprunter plusieurs routes différentes. Mais, comme on l'a vu, il ne fait après tout aucun doute que celle qu'il a le plus arpentée est celle qui partait du Dipylon pour rejoindre directement l'Académie. Notons l'utilisation, pour le verbe, du participe au datif, formulation qui constitue probablement un choix (et ne relève pas de la simple oratio variata) et a pour but d'ajouter du mouvement à la description en suggérant que le même mouvement pourra être répété par le lecteur ${ }^{44}$. À partir de ce moment, Pausanias commence la description des monuments qu'il va croiser. La formule constitue donc une balise dans la structure narrative qui correspond à un point de départ, la région du Dipylon.

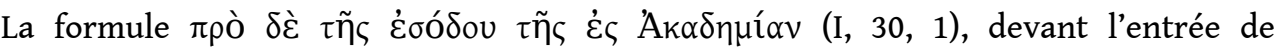
l'Académie, est une deuxième balise qui clôt une section - la route du Dipylon à l'Académie - en même temps qu'elle en ouvre une nouvelle - l'espace devant l'entrée de l'Académie.

L'espace est ainsi segmenté. Ceci nous permet d'établir une première conclusion importante : tout ce dont parle Pausanias entre ces deux balises se trouvait entre le Dipylon (ou une porte voisine) et l'espace proche de l'entrée de l'Académie, y compris les sanctuaires des Artémis Aristè et Kallistè et de Dionysos Eleuthereus. Le naos ne pouvait donc pas, si l'on s'en tient au texte de Pausanias, se trouver dans l'Académie même, ni directement devant son entrée.

Il s'agit maintenant d'analyser plus en détail la séquence comprise entre ces deux balises. Pausanias parle d'abord du péribole d'Artémis et des xoana d'Aristè et de Kallistè, puis du naos de Dionysos Eleuthereus et, enfin, des tombeaux. Certains ont considéré que l'ordre narratif de Pausanias devait correspondre à l'ordre topographique réel. Autrement dit, en descendant vers l'Académie, on aurait rencontré d'abord le temple d'Artémis puis celui de Dionysos, avant de commencer à croiser des tombeaux ${ }^{45}$. C'est négliger, cependant, une formule importante utilisée par Pausanias :

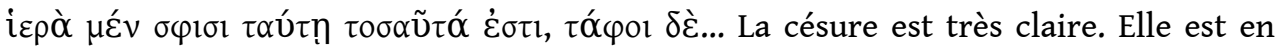
même temps une transition logique et se substitue à ce qui était ici attendu, à savoir une formule exprimant la continuité spatiale de ce qui est décrit (par exemple «non loin de », « ensuite », etc.). Or, pour quelle raison Pausanias ne donnerait-il pas d'indice de rapport géographique entre le naos de Dionysos et le tombeau de Thrasybule sinon, 
tout simplement, parce qu'il n'y en a pas? Et à l'inverse, en supposant que les tombes se soient trouvées réellement à la suite des sanctuaires lorsqu'on parcourait la voie, pourquoi Pausanias aurait-il ressenti le besoin d'utiliser une formule transitive de cet ordre? Fidèle, en fait, à la promesse de sa formule introductive, il mentionne les sanctuaires puis les tombes d'un même espace qu'il parcourt par deux fois selon une thématique différente ${ }^{46}$.

Cette hypothèse s'appuie sur d'autres passages de l'œuvre de Pausanias. Le plus évident de ce point de vue concerne les statues de l'Altis à Olympie. En effet, la description s'étend sur près de deux livres (V et VI) et Pausanias y annonce son programme de visite, sinon avec une extrême clarté, du moins en insistant sur la distinction qu'il opère. Le Périégète segmente son discours pour présenter d'abord les statues de Zeus, puis les offrandes votives qui ne sont ni dédiées à Zeus, ni liées à la gloire des hommes, et enfin les représentations de chevaux de courses et d'hommes, qu'ils fussent athlètes ou non. Outre la structuration évidente que présente ce long passage, il est remarquable que ces trois grandes séries (auxquelles on peut ajouter celle des autels de l'Altis, en $\mathrm{V}, 13,1-15,12)$ occupent en réalité un espace assez réduit de moins de 200 $\mathrm{m}^{2}$ au sein des limites de l'Altis: J. Elsner l'a bien montré, en soulignant aussi que Pausanias parcourt cet espace plusieurs fois avec différents points de vue ${ }^{47}$.

Cette structure reflète aussi les éléments qui, aux yeux du Périégète et sans doute de ses contemporains, faisaient d'olympie un site hors du commun : sa primauté rituelle (les autels), sa divinité principale (les statues de Zeus), son histoire de cultes panhelléniques (les statues de divinités) et, évidemment, les concours olympiques (les statues d'athlètes ${ }^{48}$. On ajoutera, pour s'en tenir aux statues, que Pausanias applique une hiérarchie décroissante du domaine divin (en commençant par son plus éminent représentant) vers le monde mortel, comme il le fait presque systématiquement dans toute la Périégèse.

29 Un autre passage s'inscrit dans une démarche similaire. Au septième livre de la Périégèse, Pausanias réalise le tour de force qui consiste à évoquer tout ce qu'il y a de plus remarquable en Ionie en à peine quelques lignes (VII, 5, 4-10), après un premier excursus historique où il est essentiellement question des fondations et conquêtes des villes du pays. Ce catalogue très rapide d'une région pourtant dense, et sur laquelle il y aurait beaucoup à dire, est opéré au moyen d'une structuration du discours qui se substitue à la description habituelle, relativement linéaire.

30 Après une première partie historique, Pausanias s'emploie à énumérer tout ce qui vaut la peine d'être vu en Ionie. Il commence par une liste de lieux de culte remarquables sans suivre, apparemment, d'ordre géographique simple, qui irait par exemple du nord au sud ou inversement ou qui longerait les côtes puis passerait ensuite par les îles. Au contraire, on est transporté, pour ainsi dire, d'un coin à l'autre de l'Ionie, avec même un aller-retour entre deux lieux assez éloignés (Érythrées et Priène). Après en avoir fini avec l'énumération des temples (et les quelques excursus qu'ils ont suscités), Pausanias annonce que les temples et la température ne sont pas les seules «choses appréciables » de l'Ionie (VII, 5, 10) ${ }^{49}$, amorçant ainsi le second « itinéraire ». Celui-ci lui permet d'aborder toutes les choses remarquables autres que des temples, en l'occurrence des éléments naturels du paysage, des bains, des cavernes liées à la mythologie et des tombeaux.

31 Cet apparent manque d'ordre géographique, dans ces deux énumérations successives, ne signifie pas, évidemment, qu'il n'y a aucune organisation narrative. La méthode de 
Pausanias, qu'on pourrait qualifier de "thématique », est particulièrement prégnante dans la section qui concerne les temples et préside à certains enchaînements ou regroupements par caractéristiques communes. Il s'agit moins, à vrai dire, d'une véritable structure que d'une technique narrative qui permet de masquer le caractère énumératif du passage. Ces regroupements par thème sont parfois combinés, quand cela est possible, à la proximité géographique ${ }^{50}$.

Enfin, en comparant les deux itinéraires, on remarquera que, si Pausanias ne semble pas faire ici de la cohérence géographique une priorité, du moins en apparence ${ }^{51}$, les débuts des deux parcours ioniens ("temples " et " choses remarquables») sont d'une similarité assez frappante. Dans les deux cas, il commence par Éphèse avant de se rendre dans la région de Milet puis de Colophon. Si le choix d'Éphèse comme première destination s'explique aisément par l'importance de la ville à l'époque et par son statut de capitale, le fait de passer ensuite à nouveau - et dans le même ordre - par les régions de Milet et Colophon n'est certainement pas anodin et témoigne à tout le moins du souci de Pausanias de garder une certaine cohérence dans la structure narrative.

Deux raisons, (liées l'une à l'autre), peuvent expliquer le choix de privilégier ce type de construction. Premièrement, la Périégèse de Pausanias se limite pour l'essentiel à la péninsule du Péloponnèse et à une partie de ce que l'on appelle traditionnellement la «Grèce centrale ». Au sein de ce programme de visite, la description de l'Ionie, pays d'origine probable du Périégète, ne constitue guère plus qu'un excursus inséré dans le livre qui concerne l'Achaïe et n'a d'autre but que "d'affirmer l'appartenance des concitoyens de Pausanias à la Grèce et d'inclure dans le même temps (...) les thaumata ioniens parmi les 'clés' de cette identité $»^{52}$. Pausanias veut manifestement faire bref et le passage ressemble donc, on l'a dit, à une énumération agrémentée de quelques digressions, mais sans aucune description détaillée. Ne pouvant se permettre d'utiliser le procédé habituel qui consiste à faire marcher le lecteur dans ses pas, Pausanias se doit pourtant d'organiser son discours. Son œuvre est conçue comme un récit, non comme un simple catalogue.

Ensuite, cette construction lui permet de rapprocher des monuments ou des lieux aux caractéristiques communes ou de même nature mais qui sont géographiquement éloignés les uns des autres. Ce sont, par exemple, les temples inachevés ou les bains.

Les deux exemples donnés ci-dessus, ceux des statues d'Olympie et de l'Ionie, constituent des attestations claires, dans l'œuvre de Pausanias, d'un procédé narratif qui consiste à parcourir plusieurs fois le même espace selon plusieurs points de vue différents. Certes, ces passages diffèrent de celui qui nous occupe par leur longueur, leur échelle et leur sujet. Ainsi, alors que la description de l'Ionie entend rendre compte d'une région entière, celle des abords de la route de l'Académie décrit un espace on ne peut plus linéaire, presque en une seule dimension (à ceci près que la route présente deux côtés, mais Pausanias ne précise jamais lequel des deux il évoque), et qui ne s'étend guère au-delà de $1,5 \mathrm{~km}$. Mais quelle que soit l'échelle, il faut souligner que l'auteur recourt aux mêmes procédés, à savoir le traitement répété d'un même espace selon des thématiques différentes et la hiérarchisation des thèmes, le divin étant placé toujours en premier lieu.

Reste à savoir si, dans sa description des abords de la route vers l'Académie, le Périégète respecte l'ordre topographique des monuments ou s'il opère, comme pour l'Ionie, par associations thématiques. Ou, pour reprendre l'expression de François Chamoux, si « une transition apparemment logique s'est substituée à l'énumération 
topographique habituelle... $\eta^{53}$. La meilleure hypothèse est sans doute la première, à savoir qu'au sein de chacun des deux ensembles (sanctuaires et tombeaux) construits par Pausanias, celui-ci respecte l'ordre topographique. En ce qui concerne les tombeaux, il existe des indices de nature archéologique et textuelle qui permettent d'aller dans ce sens.

Ainsi, les termes géographiques employés par Pausanias, même s'ils sont rares dans le cas de la route de l'Académie, sont significatifs. Le Périégète entame son énumération des tombeaux par celui de Thrasybule et utilise le mot $\pi \rho \tilde{\omega} \tau o v$ (1.29.3), terme qui ne peut être qu'un adverbe locatif à l'accusatif et que l'on traduira par « en premier lieu ». Il est toutefois troublant que le premier tombeau que l'on rencontre soit aussi celui d'un homme " meilleur sous tous les aspects que tous les Athéniens fameux, qu'ils aient

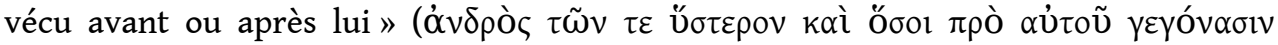

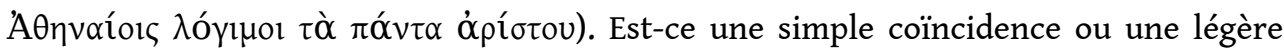
adaptation de la part du Périégète afin qu'à l'ordre des tombeaux réponde une hiérarchie (même subjective) des grands hommes athéniens ? Quoi qu'il en soit, le sens de $\pi \rho \tilde{\omega} \tau o v$ est, ici, topographique.

Pausanias le rappelle ensuite : le tombeau de Thrasybule est le premier ( $\pi \rho \tilde{\omega} \tau \circ \varsigma)$ et

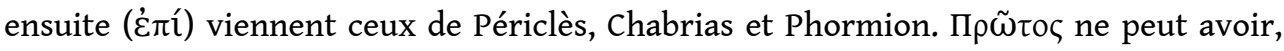
ici aussi, qu'un sens topographique, puisqu'il se rapporte directement au tombeau (et non à Thrasybule lui-même) ${ }^{54}$. On en déduira donc que Pausanias mentionne bien en premier lieu le premier tombeau qu'il rencontre - ou, à tout le moins, le premier qu'il choisit d'évoquer parmi ceux qu'il rencontre.

L'occurrence suivante du même mot est plus problématique et a suscité quelque débat. Après avoir évoqué des tombeaux individuels, Pausanias arrive aux tombeaux collectifs ou polyandreia (1.29.4). "Les premiers» qu'on a enterrés sur la route menant à l'Académie sont, dit-il, les victimes de Drabeskos. Le terme utilisé par le Périégète est $\pi \rho \tilde{\tau} \tau o l$. Certains se sont légitimement posé la question de savoir s'il s'agit d'un terme locatif ou temporel. F. Jacoby arrivait à la conclusion que la deuxième signification devait être la bonne au vu de l'organisation de la description de Pausanias ${ }^{55}$, rejetant la première qui avait été défendue par A. Brückner ${ }^{56}$. Insatisfait, W.K. Pritchett a suggéré que $\pi \rho \tilde{\omega} \tau o l$ était en fait une erreur pour $\pi \rho \tilde{\omega} \tau o v$. La confusion entre le 1 et le $v$ est en effet fréquente dans la Périégèse. Pausanias aurait utilisé le mot dans le même sens qu'en 1.29.3, c'est-à-dire au sens locatif, avec, dans le cas présent, l'idée implicite que le monument de Drabeskos était le premier du demosion sema ${ }^{57}$.

Pour le reste, la description est assez pauvre en termes géographiques, en tout cas au

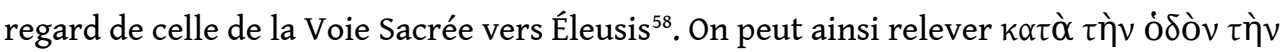

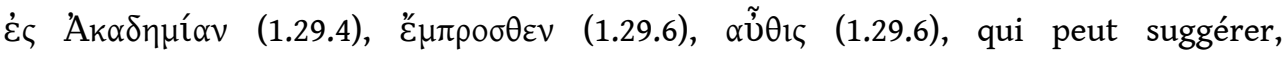
éventuellement, que Pausanias fait un petit retour en arrière, $\dot{\varepsilon} v \tau \alpha \tilde{v} \theta \alpha(1.29 .7,14,15)$,

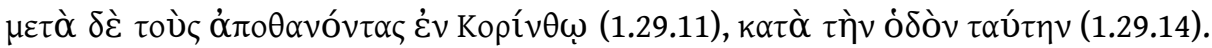

41 Ces indices, quoiqu'épars dans le texte, ont convaincu certains spécialistes que Pausanias progressait dans l'ordre topographique ${ }^{59}$. Tournons-nous à présent vers les données archéologiques.

Chr. W. Clairmont juge que seuls 13 des tombeaux mentionnés par Pausanias peuvent être identifiés à des monuments préservés dans la pierre. Parmi ces 13 identifications, 7 sont douteuses et 6 sûres, toujours d'après Chr.W. Clairmont ${ }^{60}$. Quoi qu'il en soit, la plupart de ces inscriptions ont été retrouvées en plusieurs morceaux, à des endroits 
parfois très éloignés de la route de l'Académie, et aucune d'entre elles n'a été découverte in situ ${ }^{61}$. Si l'on peut donc, en admettant que Pausanias ait relevé les monuments dans l'ordre de sa propre progression vers l'Académie, les disposer les uns après les autres sur la route, il parait difficile, voire illusoire, de les replacer à leur emplacement initial exact.

Il existe pourtant un indice important qui permet de penser que Pausanias a bien suivi l'ordre des tombeaux, même s'il ne fait aucun doute qu'il ne cite qu'une petite partie d'entre eux. Il s'agit de la découverte ${ }^{62}$, dans la région de l'Académie (à un endroit ${ }^{63}$ qui se trouve au sein du péribole de l'Académie dessiné par I. Travlos ${ }^{64}$ ), de deux stèles et un lécythe inscrits associés à des tombeaux et qui mentionnent des membres plus ou

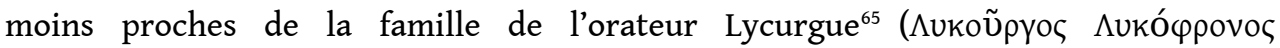
Bovtó $\left.\delta o v^{66}\right)$. Ceci laisse supposer que le tombeau de ce dernier devait se trouver, sinon à cet endroit précis, du moins à proximité, c'est-à-dire tout près de l'Académie. Or Pausanias mentionne le tombeau de Lycurgue en dernier lieu, après quoi il entreprend la description de l'entrée de l'Académie, puis de l'Académie elle-même. Ces deux informations mises côte à côte fonctionnent comme deux pièces d'un même puzzle et renforcent simultanément deux hypothèses: Lycurgue devait être inhumé dans les environs proches de la découverte archéologique et donc de l'Académie; Pausanias suivait un ordre topographique pour son énumération des tombeaux. Pourquoi, dès lors, n'aurait-il pas procédé de même avec les sanctuaires ? Le fait qu'il n'en mentionne que deux ne constitue absolument pas une raison pour penser qu'il les cite dans le désordre. Tout porte à croire, en fait, que Pausanias était assez rigoureux lorsqu'il s'agissait d'appliquer ses méthodes. Même l'excursus sur l'Ionie, malgré un itinéraire non linéaire, démontre une cohérence certaine dans les structures narratives qu'il a élaborées.

\section{La localisation du naos de Dionysos : conclusions}

Tous ces éléments nous permettent, par précisions successives, de localiser le naos de Dionysos Eleuthereus dans une zone relativement étendue mais qui peut être néanmoins définie avec précision.

Certaines indications dans le texte peuvent fonctionner comme des balises spatiales relativement précises. Elles permettent une première localisation, très générale, des temples d'Artémis Aristè et Kallistè et de Dionysos Eleuthereus : entre la région du Dipylon (comprise) et celle (non comprise) devant l'entrée de l'Académie. On précisera d'ailleurs que, puisque l'Académie était entourée d'un péribole et que même Pausanias en parle comme d'un lieu précis (et non comme d'une région), le temple de Dionysos n'a pas pu se trouver à l'Académie même.

Pausanias segmente son discours; il parcourt deux fois le même espace (celui qui sépare la région du Dipylon de l'entrée de l'Académie) selon deux angles d'approche différents. Cela signifie qu'a priori, sous réserve de nouvelles découvertes archéologiques, le temple de Dionysos peut se trouver à n'importe quel endroit dans cet espace. Si Pausanias évoque les sanctuaires avant les tombeaux, ce n'est pas pour des raisons topographiques mais " hiérarchiques ». Comme on l'a vu dans le cas des statues de l'Altis ou de l'excursus en Ionie, ce qui relève du divin est systématiquement décrit avant le reste. 
Le temple de Dionysos est mentionné après celui d'Artémis. Il est plausible que Pausanias respecte ici l'ordre topographique des monuments au sein de chaque catégorie de son discours et, par conséquent, que le temple de Dionysos ait été situé après le sanctuaire d'Artémis sur la même route. Or ce dernier a pu être localisé avec une relative précision, à environ $230 \mathrm{~m}$ au nord-ouest du Dipylon, sur la route qui conduit à l'Académie, ce qui permet de resserrer les limites de la zone d'investigation.

Il faut donc chercher le naos de Dionysos Eleuthereus entre les alentours du 11, rue Plataiôn (les fouilles d'A. Oikonomou et A. Philadelpheus) et l'entrée de l'Académie, soit dans une bande de terrain assez large (puisque la voie vers l'Académie pouvait atteindre $40 \mathrm{~m}$ de large par endroits) et longue d'environ $1,5 \mathrm{~km}$. Il paraît important ici de préciser qu'à l'inverse, rien ne permet ni d'affirmer ni d'écarter que le temple de Dionysos Eleuthereus se trouvait dans le voisinage immédiat du sanctuaire d'Artémis ${ }^{67}$.

Nous espérons que l'enquête aura apporté quelque lumière sur le dossier de la localisation des deux sanctuaires mentionnés par Pausanias dans la région du Kerameikos. L'identification du lieu de culte d'Artémis par A. Philadelpheus en sort, sinon confirmée, du moins confortée. Cette première balise posée, et en s'attachant à la structure même du discours du Périégète, nous avons pu resserrer les limites de l'espace dans lequel se trouvait le naos de Dionysos et rejeter des localisations abusives ou vagues telles que « à l'Académie » ou « dans la région de l'Académie ».

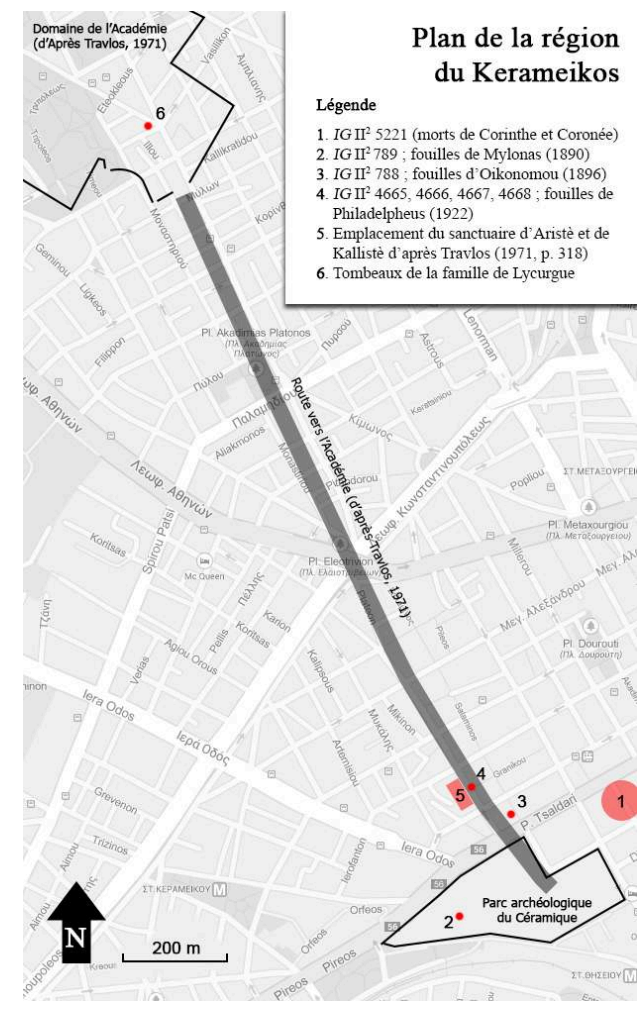

PLAN DE LA RÉgION dU KÉRAMEIKOS 


\section{BIBLIOGRAPHIE}

J. AKUJÄRVI, Researcher, Traveller, Narrator, Lund, 2005.

J. AKUJÄRVI, « Pausanias », in I.J.F. DE JONG, Space in Ancient Greek Literature : Studies in Ancient Greek Narrative, Boston / Leyde, 2012, p. 235-255.

N.T. ARRIngton, « Topographic Semantics. The Location of the Athenian Public Cemetery and Its Significance for the Nascent Democracy », Hesperia 79 (2010), p. 499-539.

L. BESCHI, D. MUSTI, Pausania, Guida della Grecia. Libro I, L'Attica. Introduzioni, testo e traduzione a cura di D. Musti. Commento a cura di L. Beschi e D. Musti, Milan, 1987.

K. BRAUN, « Der Dipylon-Brunnen B 1 . Die Funde », MDAI (A) 85 (1970), p. 129-269.

A. BRÜCKNER, « Kerameikos-Studien », MDAI (A) 35 (1910), p. 183-234.

P. BRULÉ, « Le langage des épiclèses dans le polythéisme hellénique (l'exemple de quelques divinités féminines) », Kernos 11 (1998), p. 13-34.

Fr. CHAMOUX, «La méthode historique de Pausanias », in J. BINGEN (éd.), Pausanias historien, Vandoeuvres / Genève, 1996.

Chr. W. Clairmont, Patrios Nomos. Public Burial in Athens during the $5^{\text {th }}$ and $4^{\text {th }}$ Centuries B.C., Oxford, 1983.

S.G. COLE, «Procession and Celebration at the Dionysia », in R. scodel (éd.), Theater and Society in the Classical World, Ann Arbor, 1993, p. 25-38.

E. CSAPO, W.J. SLATER, The Context of Ancient Drama, Ann Arbor, 1994.

J.K. DAVIES, Athenian Propertied Families, 600-300 B.C., Oxford, 1971.

A. von DOMASZEWKI, Der Staatsfriedhof der Athener, Heidelberg, 1917.

W. DÖRPFELD, « Funde », MDAI (A) 21 (1896), p. 458-473.

J. ELSNER, « Structuring “Greece”. Pausanias's Periegesis as a Literary Construct », in S.E. ALCOCK, J.F. CHERRY, J. ELSNER (éd.), Pausanias. Travel and Memory in Roman Greece, Oxford / New York, 2001, p. 3-20.

L.R. FARNELL, The Cults of the Greek States, vol. 5, Oxford, 1909.

W.S. FERGUSON, « Researches in Athenian and Delian Documents. I. », Klio 7 (1907), p. 213-240.

P. FOUCART, Le culte de Dionysos en Attique, Paris, 1904.

E. GRECO (éd.), Topografia di Atene. Sviluppo urbano e monumenti dalle origini al III secolo d.C. Tomo 4. Ceramico, Dipylon e Accademia, Athènes/Paestum, 2014.

G. HEDREEN, « The Return of Hephaïstos, Dionysiac Processional Ritual and the Creation of a Visual Narrative », JHS 124 (2004), p. 38-64.

W. HUTTON, Describing Greece. Landscape and Literature in the Periegesis of Pausanias, Cambridge, 2005.

F. JACOBY, «Patrios Nomos : State Burial in Athens and the Public Cemetery in the Kerameikos », JHS 64 (1944), p. 37-66. 
U. KNIGGE, Der Kerameikos von Athen. Führung durch Ausgrabungen und Geschichte, Athènes, 1988.

D. KNOEPFLER, « Sur une interprétation historique de Pausanias dans sa description du Dêmosion Sêma athénien ", in J. BINGEN (éd.), Pausanias historien, Vandoeuvres / Genève, 1996, p. 277-319.

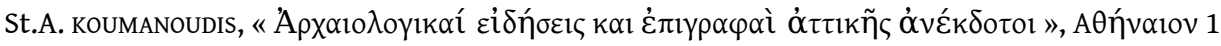
(1872), p. 395-403.

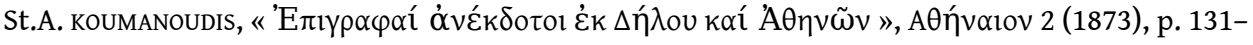
138.

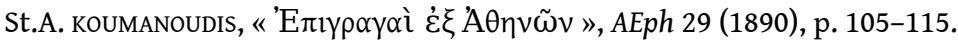

Th. MALOUTAS, A. DEFFNER, « Urbanisation et patrimoine culturel : Athènes face aux jeux olympiques de 2004 », Géocarrefour 77.4 (2002), p. 353-358.

D. MARCHIANDI, I periboli funerari nell'Attica classica : lo specchio di una 'borghesia', Athènes / Paestum, 2011.

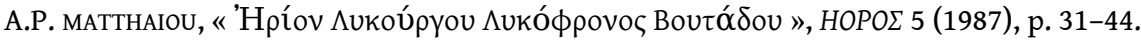

B.D. MERITT, « Greek Inscriptions », Hesperia 10 (1941), p. 38-64.

H. MöBIUS, « Neue Inschriften aus Attika und Argos », MDAI (A) 49 (1924), p. 1-16.

M. MOGGI, «L'excursus di Pausanias sulla Ionia », in J. BINGEN (éd.), Pausanias historien, Vandoeuvres / Genève, 1996, p. 79-116.

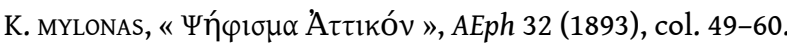

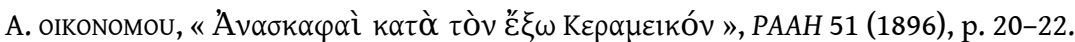

J.H. OLIVER, « Greek and Latin Inscriptions », Hesperia 10 (1941), p. 237-261.

D. PALÉOTHODOROS, « Pisistrate et Dionysos : mythes et réalités de l'érudition moderne », LEC 67 (1999), p. 321-340.

A. PAPAgeorgiou-VEnEtAs, Athens. The Ancient Heritage and the Historic Cityscape in a Modern Metropolis, Athènes, 1994.

E. PARASINOU, The Light of the Gods. The Role of Light in Archaic and Classical Greek Cult, Londres, 2000.

H.W. PARKE, Festivals of the Athenians, Londres, 1977.

R. PARKER, Polytheism and Society at Athens, Oxford, 2005.

A. PHILADELPHEUS, « Le sanctuaire d'Artémis Kallistè et l'ancienne rue de l'Académie ", BCH 51 (1927), p. 155-163.

A. PICKARD-CAMBRIDGE, The Dramatic Festivals of Athens, Oxford, 1953.

V. PIRENNE-DELFORGE, « Le lexique des lieux de culte dans la Périégèse de Pausanias ", ARG 10 (2008a), p. 143-178.

V. PIRENNE-DELFORGE, Retour à la source: Pausanias et la religion grecque, Liège, $2008 \mathrm{~b}$.

W.K. PRITCHETT, Pausanias Periegetes, Amsterdam, 1998.

P. RouSSEL, « Remarques sur le bas-relief de Kallistè », BCH 51 (1927), p. 164-169.

E. SIMON, Festivals of Attica : an Archaeological Commentary, Madison, 1983. 
Chr. SOURVINOU-INWOOD, «Something to do with Athens : Tragedy and Ritual », in R. OSBORNE,

S. HORNBLOWER, Ritual, Finance, Politics : Athenian Democratic Accounts Presented to David Lewis, Oxford, 1994, p. 269-290.

Chr. SOURVINOU-INWOOD, Tragedy and Athenian Religion, Lanham, 2003.

N. SPINETO, Dionysos a teatro. Il contesto festivo del dramma greco, Rome, 2005.

J. TRAVLOS, Bildlexikon zur Topographie des antiken Athen, Tübingen, 1971.

C. WAChSMUTH, Die Stadt Athen im Alterthum, Band 2, Leipzig 1890.

A.G. WOODHEAD, « Greek Inscriptions », Hesperia 28 (1959), p. 273-288.

\section{NOTES}

1. Pausanias, I, 29, 2-3. Toutes les traductions sont personnelles.

2. Notons qu'à aucun moment dans sa description Pausanias ne parle du quartier du Kerameikos ; il n'empêche que c'est bien cette région qu'il décrit.

3. N.T. Arrington a publié en 2010 un article qui concerne la même région avec pour objectif de localiser le demosion sema, le cimetière public d'Athènes. La présente contribution n'entend pas du tout réviser cet article ; elle vise au contraire à le compléter en prenant un angle d'approche différent : celui des sanctuaires.

4. Voir notamment MALOUTAS, DEFFNER (2002) sur cette problématique.

5. PAPAGEORGIOU-VENETAS (1994), p. $140:$ : Most of sector 1 has not been excavated ( $23.70 \mathrm{ha}$ ) and is covered by rather shabby low-rise buildings of one to three storeys. » Le secteur 1 correspond à la région archéologique comprenant l'Académie et la route qui y menait.

6. Il n'est cependant pas abusif de parler de sanctuaire, puisqu'une inscription (IG $\left.\mathrm{II}^{2}, 789\right)$, dont il sera question plus loin, évoque le hieron de Kallistè.

7. PIRENNE-DELFORGE (2008a), p. 167.

8. OІкоNOMOU (1896).

9. $I G \mathrm{II}^{2}, 788$ (voir plan, no 3).

10. DÖRPFELD (1896), p. 463.

11. PHILADELPHEUS (1927) (voir plan, no 4).

12. IG $\mathrm{II}^{2}, 4665,4668$.

13. $I G \mathrm{II}^{2}, 4667$.

14. IG $I^{2}$, 4666. ROUSSEL (1927), p. 168, a interprété la scène, assez logiquement, comme un couple venu «solliciter de la déesse une progéniture ». La torche est, en effet, un attribut commun des déesses liées à l'enfantement (PARASINOU [2000], p. 45). BRULÉ (1998), p. 25, range par ailleurs l'épiclèse Kallistè dans la sphère du féminin; on est loin ici d'une Artémis chasseresse ou guerrière (comme Sôteira par exemple). La position de la déesse, le fait qu'elle porte une torche et la présence d'un autel et d'adorants de plus petite taille sont autant d'éléments qui indiquent qu'il s'agit d'un sacrifice (PARASINOU [2000], p. 96-97). Deux reliefs comparables : LIMC Artémis 460 et 463 (IV siècle avant J.-C.).

15. Le déplacement d'un certain nombre d'inscriptions à l'Agora s'expliquerait par la récupération de blocs de la région du demosion sema pour les nouvelles constructions du centreville à partir du milieu du XIX ${ }^{\mathrm{e}}$ siècle. Voir notamment KOUMANOUdIS (1890), col. 107, PAPAGEORGIOUVENETAS (1994), p. 143 et PRITCHETT (1998), p. 2. La pierre dont il est question est d'ailleurs qualifiée «d'errante » et a été retaillée, à un certain moment, pour être remployée (WOODHEAD [1959], p. 278-279). 
16. FERGUSON (1907). Cette hypothèse, qui voulait que Kallistè et Sôteira aient été vénérées ensemble à l'Hécatéion du Céramique, avait déjà été rendue obsolète par les découvertes d'A. Philadelpheus.

17. OLIVER (1941), p. 243.

18. WOODHEAD (1959), p. 278-279.

19. Il faut encore mentionner une dédicace à Artémis d'époque hellénistique (IG $\mathrm{II}^{2}, 4689$ ), trouvée dans la région du Dipylon, que KoUMANOUDIS (1872 et 1873) a pensé pouvoir être liée au sanctuaire évoqué par Pausanias, c'est-à-dire celui des Artémis Aristè et Kallistè. En réalité, en l'absence d'épithète, cette inscription peut tout aussi bien avoir relevé d'un autre sanctuaire d'Artémis des environs. La même remarque vaut aussi pour deux fragments de plats de la fin du $\mathrm{III}^{\mathrm{e}}$ siècle avant J.-C., découverts dans le dépôt B1 du Dipylon et portant des dédicaces à Artémis (BRAUN [1970], p. 149-150, no 145 et p. 152-153, no 164). Ces découvertes attestent en tout cas la vitalité des cultes à Artémis dans la région.

20. C'est également à cette conclusion qu'était arrivé TRAVLOS (1971), p. 318. Voir plan, no 5.

21. FOUCART (1904), p. 164 ; FARNELL (1909), p. 225 ; PARKE (1977), p. 126-127 ; SIMON (1983), p. 104 ; CSAPO, SLATER (1994), p. 105 ; PALÉOTHODOROS (1999), p. 324 ; SOURVINOU-INWOOD (2003), p. 69 ; HEDREEN (2004), p.46. Ces localisations ont en commun d'être vagues et de ne pas spécifier si par Académie, on entend l'Académie elle-même ou le quartier qui se trouvait alentours.

22. Philostrate, Vie des Sophistes II, 1.

23. Malgré ce caractère relativement vague, le passage de Philostrate est important parce qu'il montre que ce transport n'était pas fait en catimini mais au grand jour et était l'occasion de premières festivités, en tout cas à l'époque de l'auteur, né vers 170 après J.-C., soit peu de temps avant la mort de Pausanias.

24. Scholie à Aristophane, Acharniens, 243a.

25. Pausanias, I, $2,5$.

26. PALEOTHODOROS (1999).

27. C'est le cas des représentations théâtrales. Cf. PICKARD-CAMBRIDGE (1953), p. 73. Les différentes processions ont-elles subi des changements significatifs au cours du temps ? C'est plus difficile à dire. Cependant, PICKARD-CAMBRIDGE (1953), p. 58, n. 3, estime que ce que décrit Philostrate dans le passage cité plus haut (Vies des Sophistes 2.1) est une "perversion tardive d'une fête qui avait perdu son sens ». Il est en tout cas très probable que cette pratique n'existait pas, ou du moins pas sous cette forme, aux premiers siècles des célébrations.

28. Pausanias, I, 29, 2.

29. COLE (1993), p. 27, dit que la statue venait d'Éleuthères, « home of Pegasos ». En dehors du fait que Pegasos venait effectivement d'Éleuthères, il n'y a à notre connaissance aucun document permettant d'étayer cette théorie.

30. Terme des décrets éphébiques $\left(I G \mathrm{II}^{2}, 1006,1008,1011,1032\right)$ qui remontent à la fin du $\mathrm{II}^{\mathrm{e}}$ siècle (de 127 à 106 av. J.-C.).

31. Voir, par exemple, CSAPO, SLATER (1994), p. 105.

32. C'est ce qu'affirment, avec des arguments convaincants, SOURVINOU-INWOOD (1994), p. 278-288,

et SPINETO (2005), p. 221-222. PARKER (2005), p. 318, reprend cette interprétation dans sa description des processions.

33. Pour une description détaillée de l'ensemble des processions, voir SPINETo (2005), p. 217-230. Voir aussi PICKARD-CAMBRIDGE (1953), p. 55-103.

34. ARRINGTON (2010), p. 528. D'après lui, le demosion sema se trouvait à $200 \mathrm{~m}$ environ au nordouest du Dipylon (p. 524).

35. KNIGGE (1988), p. 41-42.

36. Diodore de Sicile, XXVIII, 7 ; Tite-Live, XXXI, 24, 17-18.

37. Plutarque, Vie de Sylla, 12. 
38. DOMASZEWSKI (1917), p. 4, 6, 18 ; JACOBY (1944), p. 40, n. 12 : « Again, Pausanias himself does not mention either the devastation or the restoration; but if he tells us e.g. in $\S 11$ that the same stele

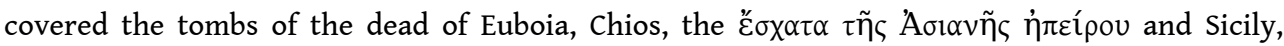
hardly another explanation than a restoration can be found ».

39. ARRINGTON (2010), p.515, reprend les deux hypothèses pour expliquer l'absence de tout monument dans la description de Pausanias avant le péribole d'Artémis: "The fact that Pausanias is silent about polyandria up to this point in his route, however, need not indicate that the public graves began only after the shrine. He may have taken a cross-street from the old Academy Road to the shrine, or his silence may be attribuable to the fact that the graves close to the city walls had been covered up in the 4th century ".

40. En particulier s'il a pris la « Old Academy Road » qui devait être un haut lieu de la mémoire aristocratique athénienne d'après ARRINGTON (2010).

41. HUTTON (2005), p. 143-144, met en garde contre une interprétation topographique trop rapide, basée sur l'ordre des mots, du texte de Pausanias : « ... Pausanias usually, if not always, chooses the order of words for stylistic effect rather than topographical clarity ».

42. PIRENNE-DELFORGE (2008b), p. 243 : «Une structuration s'amorce, bien servie par l'ordre des mots - volontaire ou non... ".

43. BESCHI, MUSTI (1987), p. 373 : «la frase indica la linea programmatica delle scelte di Pausania... ».

44. AKUJÄRVI (2005), p. 147-148. Cette utilisation du participe au datif par Pausanias est cependant relativement fréquente.

45. C'est encore le cas, récemment, de D. Marchiandi, qui pense que les tombes publiques du demosion sema proprement dit ne commençaient qu'après le naos de Dionysos Eleuthereus, tout en admettant que celui-ci reste à découvrir (MARCHIANDI [2011], p. 285 et GRECO [2014], p. 1439-1440).

46. D. Knoepfler adopte, semble-t-il, la même interprétation. Cf. KNOEPFLER (1996), p. 282, n. 15 : «... le Périégète décrit ici les choses katò ع̌ $\theta$ vos, d'abord les hiéra $(29,2)$, puis les taphoi (29, 3 sqq.) : le sanctuaire ne marque pas le point de départ de sa visite de la nécropole... ».

47. ELSNER (2001), p. 10-11. Cette organisation de la narration a également été décrite par AKUJ̈̈RVI (2012), p. 246, qui souligne la combinaison entre une organisation topographique d'une part, et « systématique » d'autre part.

48. ELSNER (2001), p. 17.

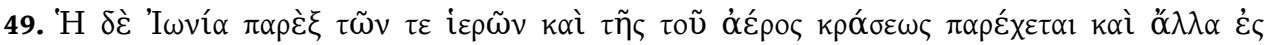
бчүүрачท́... On notera la ressemblance fonctionnelle sinon formelle de cette formule avec celle que Pausanias utilise pour faire la transition, au Kerameikos, entre temples et tombeaux (I, 29, 3). Les deux formules ont en tout cas en commun de lancer une nouvelle visite, sous un angle différent, d'une région déjà parcourue.

50. Ainsi que l'a fait remarquer AKÜJARVI (2012), p. 245-246.

51. L'ordre géographique n'est très probablement pas indifférent. Pour la partie historique sur l'Ionie qui précède, MAURO MOGGI (1996), p. 84, note que Pausanias distingue le continent des îles et suit un itinéraire sud-nord, avec pour exception Éphèse, mentionnée avant Priène, concession aux deux traditions concurrentes d'Hérodote et Phérécyde.

52. PIRENNE-DELFORGE (2008b), p. 172.

53. CHAMOUX (1996), p. 53.

54. À moins, bien sûr, que Pausanias n'ait voulu dire qu'il s'agit du tombeau le plus remarquable. Mais dans ce cas on s'attendrait à recevoir au moins un début de justification, comme dans le cas de l'Artémision d'Éphèse : « en raison de sa taille et de ses richesses » (VII, 5, 3).

55. JАСОВY (1944), p. 53-54, n. $77:$ : $\pi \rho \tilde{\omega} \tau$ or is, in fact, purely temporal ».

56. BRÜCKNER (1910), p. 197. 
57. PRITCHETT (1998), p. 39-40. Cela implique, pour W.K. Pritchett, que rien de ce qui précède dans le discours de Pausanias ne faisait partie du demosion sema. Pour rappel, ARRINGTON (2010), p. 528, pense quant à lui que le naos de Dionysos avait de fortes chances de s'y trouver. Peut-être faut-il alors comprendre $\pi \rho \tilde{\omega} \tau o v$ (en admettant que l'hypothèse de W.K. Pritchett soit correcte) dans le sens de " premier des tombeaux collectifs".

58. Les formules ne sont pas tellement plus nombreuses, mais elles sont plus précises, ce qui s'explique aisément par l'étendue de la zone décrite et sa densité d'occupation. Pausanias peut cette fois utiliser des éléments naturels, comme le Céphise (I, 37, 3 et 4) ou les Rheitoi (I, 38, 1), comme points de repères commodes pour la compréhension de sa progression. La linéarité de celle-ci, dans un espace à la fonction comparable à celui parcouru vers l'Académie, laisse à croire que le Périégète est tout aussi linéaire sur la route vers l'Académie.

59. Notamment Fr. Chamoux (KNOEPfLER [1996], p. 315-316) et PRITCHETT (1998), p. 40.

60. CLAIRMONT (1983), p. 33.

61. PRITCHETT (1998), p. 1. C'est par exemple le cas de $I G I^{2}$, 5221, découverte en contexte moderne à un endroit (voir plan, no 1) probablement assez éloigné de son lieu d'origine puisque Pausanias la mentionne assez tard dans son parcours (I, 29, 11).

62. L'importance topographique et pas seulement prosopographique de cette découverte a été soulignée et discutée par MATTHAiou (1987).

63. À l'intersection des rues Vassilikôn et Kratylou (plan, no 6). Voir A $\Delta 34$ (1979) B' 1, Xpovıkó, p. 18-20.

64. TRAVLOS (1971), p. 318, fig. 417.

65. Si Lycurgue n'est pas mentionné directement, il apparaît cependant sur le lécythe, dans le nom d'un de ses trois fils. La stèle A mentionne quant à elle le père de l'orateur et la stèle $\mathrm{B}$ un cousin du père et ses deux fils.

66. Sur ce personnage et sa famille, voir DAVIES (1971), p. 348-353, table IV.

67. Selon CLAirmont (1983), p. 30, les tombeaux de Thrasybule, Périclès, Chabrias, Phormion doivent être placés entre le Dipylon et le temple d'Artémis Aristè et Kallistè. Ceci suggère qu'il adopte un point de vue similaire au nôtre, à savoir que Pausanias a donné à son discours une armature thématique et non topographique. Pourtant, sur ses cartes, Chr.W. Clairmont situe le naos de Dionysos (avec un point d'interrogation) juste après celui d'Artémis (vol. 2, fig. 1 et suivantes), sans en donner la raison.

\section{RÉSUMÉS}

Le naos de Dionysos Eleuthereus, croisé par Pausanias sur la route menant du Dipylon à l'Académie, n'a jamais pu être décrit faute de découverte qui pourrait lui être associée de manière sûre. Sa localisation varie selon les auteurs, qui le situent à différents endroits et de manière plus ou moins vague, sans qu'aucun argument soit déterminant. Il est cependant possible de resserrer les limites de l'espace d'investigation par une analyse attentive du passage du Périégète qui concerne cette région. L'auteur ne suit vraisemblablement pas un ordre topographique strict, mais structure sa description en deux volets et parcourt le même espace deux fois selon des thèmes différents: d'abord les sanctuaires, puis les tombeaux. L'autre sanctuaire mentionné par Pausanias dans la région, celui des Artémis Aristè et Kallistè, peut 
fonctionner ici comme une balise spatiale et narrative. Or sa localisation, si elle n'a guère été discutée depuis près d'un siècle, est assez sûre.

The naos of Dionysos Eleuthereus mentioned by Pausanias on the road between the Dipylon and the Academy has never been described because of the lack of findings that could be safely associated with it. Its location varies depending on the scholars, who place it in various spots, sometimes vaguely, without any decisive argument. Nevertheless, it is possible to narrow the investigation area by carefully analyzing the passage of the text that concerns this region. The author probably does not follow a strict topographical order but organizes his description in two parts and walks twice through the same space with different themes in mind: first the sanctuaries, then the graves. The other sanctuary mentioned by Pausanias in the region, that of Artemis Ariste and Kalliste, may be considered here as a spatial and narrative marker. Although it has been little discussed for almost a century, its location is quite sure.

\section{AUTEUR \\ JEAN VANDEN BROECK-PARANT}

CReA-Patrimoine, Université libre de Bruxelles

Av. Franklin D. Roosevelt 50, CP 175

1050 Bruxelles

jevdbroe@ulb.ac.be 